\title{
Polymorphism of Myostatin (MSTN) Promoter Gene and its Association with Growth and Muscling Traits in Bali Cattle
}

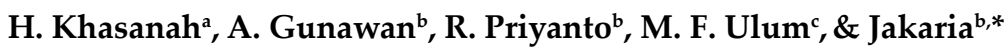 \\ astudy Program of Animal Production and Technology, Faculty of Animal Science, Graduate School, \\ Bogor Agricultural University \\ bDepartment of Animal Production and Technology, Faculty of Animal Science, Bogor Agricultural University \\ 'Department of Clinic, Reproduction and Pathology, Faculty of Veterinary Medicine, Bogor Agricultural University \\ Jalan Agatis, Kampus IPB Darmaga Bogor 16680, Indonesia \\ (Received 12-05-2016; Reviewed 17-06-2016; Accepted 19-07-2016)
}

\begin{abstract}
Myostatin (MSTN) gene plays a key role in skletal muscle homeostasis such as inducing muscle athrophy, poliferation of myoblast, increasing ubiquitin-proteasomal, downregulating IGF pathway, and glucolysis. Myostatin gene expression is controled by $\mathrm{CpG}$ island located in promoter region. The objectives of this research were to identify polymorphism of MSTN promoter gene and to associate the polymorphism of SNP with growth and muscling traits in Bali cattle. A total of 48 Bali cattle from BPTU-HMT Bali island was screened to identify genetic polymorphisms in MSTN promoter region using sequencing method. The growth and muscling traits were measured at 12 months of age. The muscling traits were evaluated using ultrasound console with linear transducer having frequency $6.5 \mathrm{~Hz}$ and scaning we conducted at $130 \mathrm{~mm}$ in deep. Analysis of polymorphism was conducted by using PopGen 1.32 software. The association of MSTN with growth and muscling traits were analyzed by using General Linear Model (GLM) procedure. This result showed that a total of 20 polymorphic SNPs (seven SNPs in CpG island) were detected in this region. Although, only $3 \mathrm{SNPs}$ (g.-8078C $>\mathrm{T}$, g.-7996G $>\mathrm{C}$, and g.-7930A $>$ G) had equilibrium condition in Hardy-Weinberg analysis. The association result showed that 2 SNPs (g.-7799T $>C$ and g. $-7941 \mathrm{C}>\mathrm{T}$ ) were significantly associated with intramuscular fat percentage $(P \leq 0.05)$ in Bali cattle. Although the 2 SNPs were nominally significant at nominal $P \leq 0.05$ threshold, they were not significant after Bonferroni correction for multiple testing. It could be concluded that MSTN promoter gene was polymorphic in Bali cattle and there were 2 SNPs associated with carcass quality.
\end{abstract}

Key words: Bali cattle, CpG island, myostatin gene, polymorphism, SNP

\section{ABSTRAK}

Gen myostatin (MSTN) berperan dalam homeostasis otot rangka seperti induksi athrophy otot, myosblast poliferasi, peningkatan ubiquitin-proteasomal, penurunan pathway IGF dan glikolisis. Ekspresi gen myostatin (MSTN) dikontrol oleh $C p G$ island yang berlokasi di promoter. Tujuan dari penelitian ini adalah mengidentifikasi polimorfisme dari promoter gen MSTN dan mengasosiasikan polimorfisme dari SNP dengan sifat pertumbuhan dan perdagingan pada sapi Bali. Sebanyak 48 sapi Bali yang berasal dari BPTU-HMT pulau Bali diidentifikasi polimorfisme dari gen MSTN bagian promoter menggunakan metode sekuensing. Sifat pertumbuhan dan perdagingan diukur pada umur 12 bulan. Sifat perdagingan diukur menggunakan ultrasonografi dengan linier transduser dan pembacaan pada frekuensi $6,5 \mathrm{~Hz}$ dan kedalaman $130 \mathrm{~mm}$. Analisis polimorfisme dilakukan menggunakan software Popgen 1.32. Asosiasi gen MSTN dengan sifat pertumbuhan dan perdagingan dianalisis dengan menggunakan prosedur General Linear Model (GLM). Hasil penelitian ini menunjukkan sebanyak 20 SNP yang polimorfik ditemukan di daerah ini (7 SNP berada pada posisi CpG island). Namun, hanya ada 3 SNP, yaitu g.-8078C $>$ T, g.-7996G $>\mathrm{C}$ and g.-7930A $>\mathrm{G}$ yang ditemukan dalam keadaan seimbang berdasarkan analisis Hardy-Weinberg. Hasil asosiasi ditemukan bahwa SNP g.-7799T $>\mathrm{C}$ dan g.-7941C $>\mathrm{T}$ berasosiasi nyata dengan sifat persentase lemak intramuskuler $(P \leq 0,05)$. Meskipun 2 SNP tersebut signifikan pada $\mathrm{P} \leq 0,05$, namun tidak signifikan setelah dikoreksi Bonferroni untuk uji berganda. Dapat disimpulkan bahwa gen promotor MSTN polimorfik pada sapi bali dan terdapat 2 SNP yang berasosiasi dengan sifat kualitas karkas.

Kata kunci: CpG island, gen myostatin, polimorfisme, sapi Bali, SNP 


\section{INTRODUCTION}

The myostatin (MSTN) gene is well-known as growth and differentiation factor 8 (GDF8) that is belonging to a member of transforming growth factor $\beta$ (TGF- $\beta$ super family). This gene consists of 3 exons and 2 introns (Kambadur et al., 2004). Myostatin gene has been located close to the centromere of bovine chromosome 2 (BTA 2) and encoded 375 amino acids then produce myostatin protein with $26 \mathrm{kDa}$ molecular weight (Kambaduret al., 2004). Myostatin plays a key role in skletal muscle homeostasis such as inducing muscle athrophy, poliferation of myoblast, increasing ubiquitin-proteasomal, down regulating IGF pathway, and glukolisis (Elliott et al., 2012). The function of MSTN gene was an inhibitor (negative regulator) of proliferation and differentiation of cell cycle during myogenesis in embryonic and adult cell (Miyake et al., 2010). Kambadur et al. (2004) have identified that absence of myostatin affected increasing of skeletal muscle mass. This increment is due to a combination of hyperplasia (increasing muscle number) and hypertrophy (increasing muscle size).

Based on gene structure, MSTN has a CpG island, a region with repetitive of GC sequences that $70 \%-80 \%$ find in promoter of gene (Illingworth et al., 2010). The distribution of transcription initiation is usually over a region of 50-100 bp and there are appearance of the CpG island and lack of TATA box (Carninci et al., 2006). The function of genome platforms for regulating transcription associated with promoter especially in a CpG island which has a role as genomic platforms to regulate transcription (Deaton et al., 2011). In addition, the CpG island was common for methylation among the region of the promoter attributing of chromatin condense and gene silencing (Sellner et al., 2007). Methylation of imprinted gene can increase or decrease the level of transcription, depending on a positive (suppressor) or negative (repressor) regulatory (Smith \& Meissner, 2013). In vertebrates, the CpG island is distinct owing to their lack of DNA methylation and absences of CPG deficiency (Deaton et al., 2011). Mutation in CpG island of MSTN gene could change the regulation of expression via the generated $\mathrm{CpG}$ island and/or changed target sites for transcriptional regulator (Doherty et al., 2014). Mutation in MSTN gene in cattle showed different characteristics such as increasing of birth weight, higher muscling, faster growing, hyperplasia and hypertrophy in muscle (Kambadur et al., 2004). Previous research has identified MSTN gene in cattle intensively such as in Hanwoo cattle (Han et al., 2012), Qinchuan cattle (Zhang et al., 2007), Nellore cattle (Grisolia et al., 2009), Angus cattle (Gill et al., 2009), and Marchigiana cattle (Sarti et al., 2014).

Bali cattle (Bos javanicus) is one of Indonesian origin genetic resources that domesticated from bull (Bibos banteng) (Martojo et al., 2012). Bali cattle have potential to be beef cattle because well adapted in harsh environment, able to grow in marginal feed condition, high fertility and conception rate than other breeds (Purwantara et al., 2012). However, the utilization of Bali cattle is not optimal yet to produce meat in high quantity and quality. Improving Bali cattle quality by selection was conducted based on phenotypics data (conventional method) which has susceptibility that their environment impact. Selection using marker assisted selection (MAS) could be one of promising method for selection in cattle because it is more accurate, effective and effisien (Goddard \& Hayes, 2007; Gorjanc et al., 2015). Therefore, improving Bali cattle genetic quality based on MAS using potential gene such as MSTN gene needs to be done on Bali cattle. The objective of this research was to identify the Single Nucleotide Polymorphism (SNP) of the MSTN gene in Bali cattle related to growth and muscling traits using direct sequencing method. The analyses of genotype and allele frequency were performed to elucidate polymorphism of this gene in Bali cattle. Association of MSTN with growth and muscling traits were also performed to identify significant SNP and its candidate for the genetic marker.

\section{MATERIALS AND METHODS}

\section{Animal and Phenotypic Data Source}

A total 48 of Bali cattle were used (12-15 month of ages) in this study that consisted of 24 heifers and 24 steers from BPTU-HMT Denpasar, Bali Province. All of samples were risen in same paddock and feeding management. Each cattle was fed with grass (Pennisetum purpureum and Phaspalum notatum) in the amount of $10 \%$ of body weight and feed concentrate as much as $1 \%$ of body weight. The phenotypic variables that observed were growth traits including birth weight (BW), weaning weight (WW), yearling weight (YW), average daily gain (ADG), chest circumference (CC), body length (BL), and shoulder height $(\mathrm{SH})$. Growth traits were measured based on BSN (2015). The muscling traits were evaluated using ultrasound console with linier transducer having frequency $6.5 \mathrm{~Hz}$ and scning were conducted with deep of $130 \mathrm{~mm}$ at transversal and longitudinal views. The muscling traits of ultrasound longissimus dorsi thickness (LDT), ultrasound back fat thickness (BFT), ultrasound rump thickness (RT), ultrasound rump fat thickness (RFT), ultrasound marbling score (MS) and Intramuscular fat percentage (PIMF) were assessed in this study. The measurement of LDT and BFT were carried out on the $12^{\text {th }}-13^{\text {rd }}$ ribs, two third from medial to lateral side models (Gupta et al., 2013; Melendez \& Marchello, 2014) (Figure 1). The variables RT and RFT were measured between ileum and ischium (Silva et al., 2012) modified. In brief, the measurement of MS carried out according to AUS MET and MSA marbling reference standard. The percentage of IMF was carried out according to Deaton et al. (2000) on $12^{\text {th }}-13^{\text {rd }}$ ribs with region of interest by $30 \times 30 \mathrm{~mm}$. The image results were analyzed by using Image-J NIH software (ImageJ $₫$, NIH, USA) (Figure 1). The general description of growth and muscling traits are shown in Table 1.

\section{Genome Extraction and Amplification}

Approximately $10 \mathrm{~mL}$ blood per cattle was collected aseptically from the jugular vein and kept in a 
Table 1. Overall growth and muscling traits in Bali cattle

\begin{tabular}{lcrrrc}
\hline \multicolumn{1}{c}{ Traits } & $\mathrm{n}$ & Means & \multicolumn{1}{c}{ SD } & \multicolumn{1}{c}{ Max } & Min \\
\hline Birth weight (Kg) & 48 & 17.19 & 1.32 & 22.00 & 15.00 \\
Weaning weight (Kg) & 48 & 57.05 & 10.91 & 81.02 & 40.57 \\
Yearling weight(Kg) & 48 & 88.10 & 19.44 & 128.65 & 59.71 \\
$\begin{array}{l}\text { Average daily gain } \\
\text { (Kg) }\end{array}$ & 48 & 0.18 & 0.08 & 0.30 & -0.05 \\
$\begin{array}{l}\text { Shoulder height (cm) } \\
\text { Body length (cm) }\end{array}$ & 48 & 91.91 & 5.48 & 106.00 & 82.00 \\
$\begin{array}{l}\text { Chest circumtance } \\
\text { (cm) }\end{array}$ & 48 & 108.91 & 9.18 & 133.00 & 94.00 \\
$\begin{array}{l}\text { Longissimus dorci } \\
\text { thickness (mm) }\end{array}$ & 31 & 30.02 & 4.88 & 38.52 & 22.26 \\
$\begin{array}{l}\text { Back fat thickness } \\
\text { (mm) }\end{array}$ & 31 & 1.27 & 0.29 & 2.04 & 0.83 \\
$\begin{array}{l}\text { Rump thickness } \\
\text { (mm) }\end{array}$ & 31 & 36.40 & 4.82 & 46.35 & 28.67 \\
$\begin{array}{l}\text { Rump fat thickness } \\
\text { (mm) }\end{array}$ & 31 & 0.94 & 0.27 & 1.60 & 0.46 \\
$\begin{array}{l}\text { Marbling score } \\
\begin{array}{l}\text { Percentage of IMF } \\
\text { (\%) }\end{array}\end{array}$ & 31 & 1.87 & 0.88 & 3.40 & 0.00 \\
\hline
\end{tabular}

tube containing anticoagulant of EDTA under temperature of $4^{\circ} \mathrm{C}$. Genome extracted by using genomic DNA mini Kit (GeneAid DNA Ltd, Taiwan). The quality of total genome extractionswas performed by $1 \%$ agarose gel electrophoresis and was checked using spectrophotometry. The pairs of primer were used to amplify part of MSTN promoter gene. The forward primer: 5'-CCAACTATCCACCAGTAA-3' and the reverse primer: 5'-ACGACCAACCCTAACC-'3 were designed according to bovine MSTN gene (GenBank: AF348479.1) by using primer designing tool program (http://www. ncbi.nlm.nih.gov/tools/primer-blast/) and primer stat program (http://www.bioinformatics.org/sms2/ pcr_primer_stats.html). PCR reaction of MSTN gene was $50 \mu \mathrm{L}$ consisted of $2 \mu \mathrm{L}$ DNA sample, $22.6 \mu \mathrm{L}$ distilled water, $0.2 \mu \mathrm{L}$ forward and $0.2 \mu \mathrm{L}$ reverse primers, and $25 \mu \mathrm{L}$ GoTaq Promega Green MM. The PCR conducted in GeneAmp® PCR System 9700 Applied Biosystem Thermalcycler. Amplification condition consisted of predenaturation at $95^{\circ} \mathrm{C} 5 \mathrm{~min}$ followed by 35 cycles of denaturation at $95^{\circ} \mathrm{C}$ for $10 \mathrm{~s}$, amplification at $63^{\circ} \mathrm{C}$ for $20 \mathrm{~s}$, extention at $72^{\circ} \mathrm{C}$ for $30 \mathrm{~s}$, and a final extension at $72^{\circ} \mathrm{C}$ for $5 \mathrm{~min}$. The DNA amplification products were checked on $1.5 \%$ agarose gels in $0.5 \times$ TBE running buffer and stained with EtBr then were visualized in UV trans-illuminator.

\section{SNP Identification}

Sequencing was performed for all of Bali cattle samples to define SNP in MSTN promoter region. Forward and reverse primer fragments were sequenced using sequencer machine (ABI Prims 3100-Avant Genetic Analyzer) in $1^{\text {st }}$ Base Selangor, Malaysia. The sequencing results were aligned using MEGA software (Tamura et al., 2011) to establish SNP. The BLAST (Basic Local

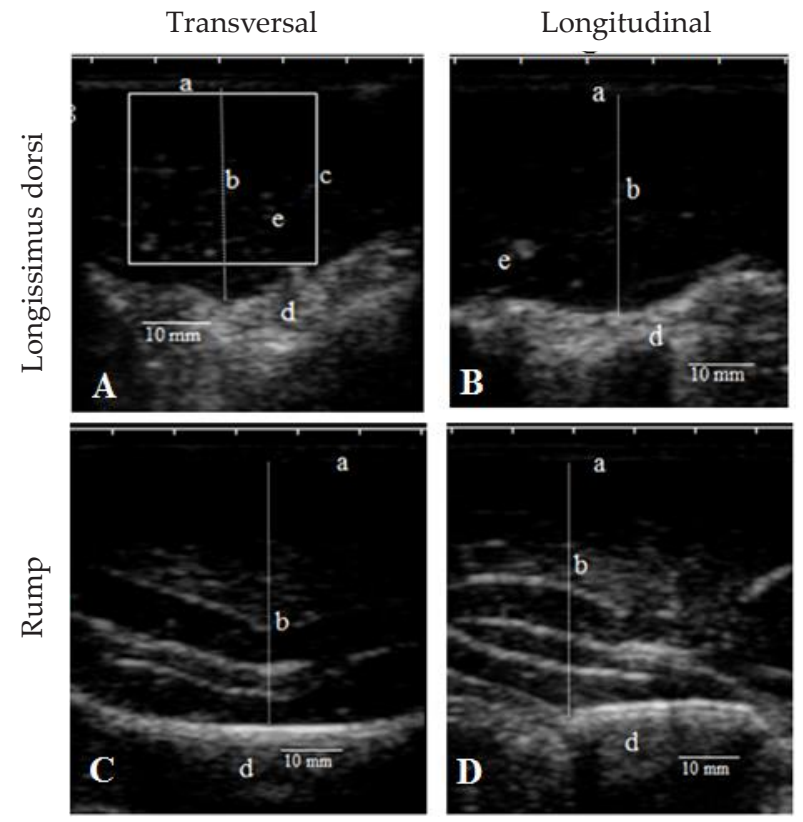

Figure 1. Ultrasound of muscling traits in Bali cattle (A) and (B) longissimus dorsi at 12 th-13rd rib; (C) and (D) rump; (a) fat thickness, (b) muscle thickness, (c) region of interest of Intramuscular fat percentage (PIMF), (d) bone.

Alignment Search Tool) program was used to search reference and homologous sequences in GenBank database.

\section{Data Analysis}

The genotypic and allelic frequencies from SNP, heterozygosity and Hardy-Weinberg equilibrium were calculated using PopGen program (Yeh et al., 1999). The association of MSTN gene and growth trait was analyzed by ANOVA PROC GLM and Duncan multiple ranget test (DMRT) procedure of SAS (SAS Inst., 2008). Furthermore, we also conducted Bonferroni correction for multiple testing. The statistical model used as follows the formulas below: $Y_{\mathrm{ijk}}=\mu+\alpha_{\mathrm{i}}+\beta_{\mathrm{j}}+\varepsilon_{\mathrm{ij}}$

where $Y_{i j k}$ is the mean value of the trait; $\mu$ is the general mean; $\alpha_{i}$ is the fixed effect of MSTN genotype $(\mathrm{i}=1,2,3)$; $\beta_{\mathrm{j}}$ is the fixed effect of $\operatorname{sex}(\mathrm{j}=1,2)$; $\varepsilon_{\mathrm{ij}}$ is the random error.

\section{RESULTS}

\section{Polymorphism of MSTN Promoter Gene in Bali Cattle}

Result of PCR amplification consisting of $535 \mathrm{bp}$ PCR products with 100 bpmarker was showed in Figure 2. Bali cattle sequences of MSTN gene were aligned with GenBank AF348479 by MEGA blast tools resulting 20 polymorphic SNPs in the promoter region. The polymorphisms of MSTN promoter gene were caused by transversion mutation at g. $-8350 \mathrm{C}>\mathrm{T}$, g. $-8310 \mathrm{~A}>\mathrm{C}$, g.-8299G $>A, \quad$ g. $-8283 A>G, \quad$ g. $-8216 G>A, \quad$ g. $-8205 A>G$, g.-8168A $>$ G, g.-8109T $>G, \quad$ g. $-8078 C>T, \quad$ g.-8077G $>A$, g. $-8029 \mathrm{~T}>\mathrm{C}, \quad$ g. $-8028 \mathrm{~A}>\mathrm{G}$, g. $-8016 \mathrm{C}>\mathrm{T}$, g. $-7799 \mathrm{~T}>\mathrm{C}$, g. 


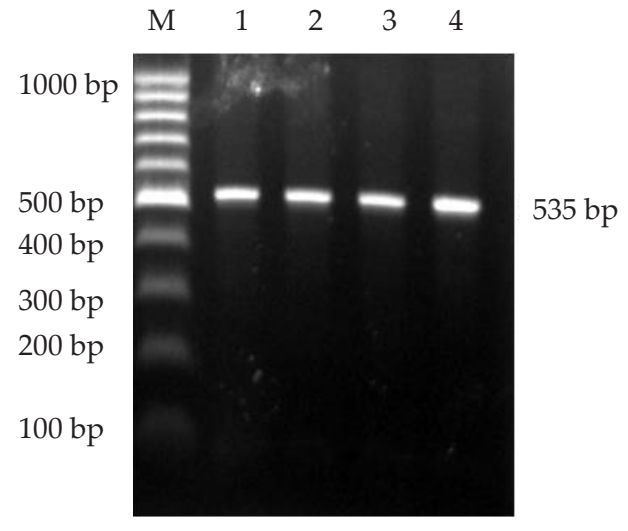

Figure 2. Amplification myostatin gene promoter in Bali cattle (line 1-4; 535 bp); M: 100bp ladder.

7953C > T, g. $-7941 \mathrm{C}>\mathrm{T}$, g. $-7930 \mathrm{~A}>\mathrm{G}$, g. $-7905 \mathrm{~T}>\mathrm{C}$, and transition mutation at g. $-7996 \mathrm{G}>\mathrm{C}$ and g.-7942C $>\mathrm{G}$ (Figure $3)$. The frequencies of genotype and allele were showed in Table 2. Mostly, SNPs had 3 genotypes except SNP g.8109T $>$ G andg.-7905T $>C$, there were only homozygote genotype. The highest heterozygosity was found in SNP g.-7996G $>$ C. In addition, the smallest heterozigosity were g.-8109T $>\mathrm{G}$ and g.-7905T $>C$ having 0.000 value due to no heterozygote genotype found (Table 2). This re- search found that only 3 SNP in equilibrium condition, they were g. $-8078 \mathrm{C}>\mathrm{T}$, g.-7996G $>\mathrm{C}$, and g. $-7930 \mathrm{~A}>\mathrm{G}$ (Table 2).

\section{CpG Island Prediction in MSTN Promoter Gene}

The sequence target of amplification was in promoter region which had $\mathrm{CpG}$ island (prediction using http://www.urogene.org/methprimer/). This prediction using criteria with minimum sequence length was $>100 \mathrm{bp}$, GC percentageis $>50 \%$ and Obs/Exp ratio was $>0.6$. Seven mutation was found in $\mathrm{CpG}$ island region, they were g.-7799T $>\mathrm{C}$, g.-8078C $>\mathrm{T}$, g.-8077G $>\mathrm{A}$, g.-8029T>C, g.-8028A $>$ G, g. $-8016 C>T$, g. $-8016 C>T$, g.$7799 \mathrm{~T}>\mathrm{C}$. This mutation might be affected the absences of CpG island in Bali cattle sequence (Figure 4).

\section{Association Analysis}

The association analysis showed that no growth traits were significantly associated with SNPs in MSTN promoter gene in Bali cattle $(\mathrm{P}<0.05)$ (Table 3$)$. In this result also showed that SNPs had no association with muscling traits, except 2 SNPs (g.-7799T $>C$ and g.7941C > T) which had significant effect on PIMF $(\mathrm{P}<0.05)$ (Table 4). In the SNP g.-7799T $>$ C, TT genotype has higher PIMF than CT and CC genotype in the SNP g.$7941 \mathrm{C}>\mathrm{T}$ has higher PIMF than TT. Although the 2 SNPs

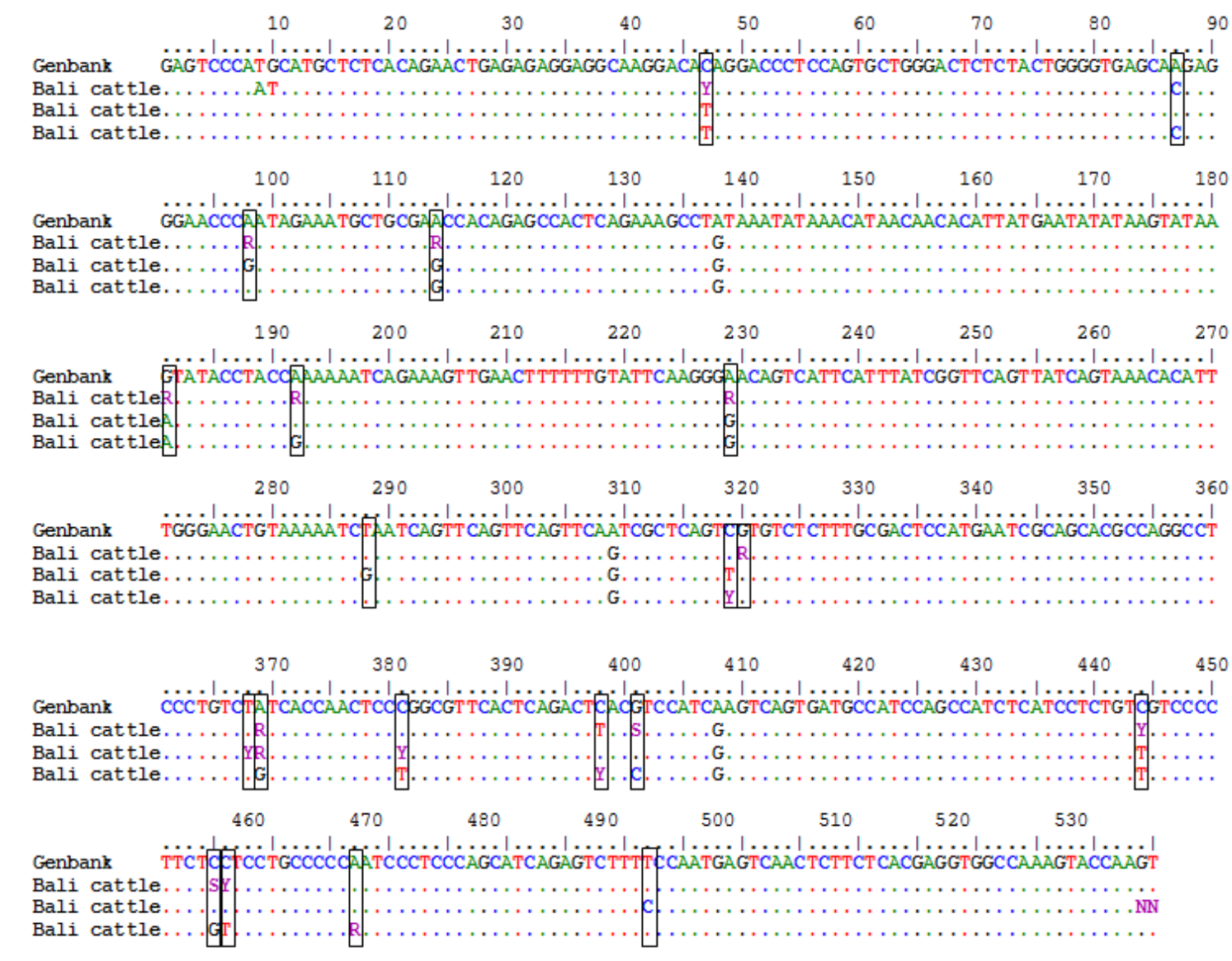

Figure 3. SNPs in myostatin promoter gene in Bali cattle 
are nominally significant at nominal $\mathrm{P} \leq 0.05$ threshold, they are not significant after Bonferroni correction for multiple testing.

\section{DISCUSSION}

\section{Polymorphism of Myostatin Promoter Gene}

The SNPs in this research was polymorphic which represented by allele frequency lower than 0.99 or allel frequency higher than 0.01 (Nei \& Kumar, 2000).
Heterozygosity value showed that all of SNPs in low diversity condition (Ho $\leq 0.5)$ (Allendrof et al., 2013). Based on chi-square $\left(\chi^{2}\right)$ analyses, only 3 SNPs in Bali cattle were in equilibrium condition (SNPs g.-8078C $>\mathrm{T}$, g.-7996G>C, and g.-7930A>G). The factors which influenced Hardy-Weinberg equilibrium are non-random mating, selection, mutation, migration and genetic drift (Allendrof et al., 2013). Disequilibrium in Bali cattle genetic diversity might be caused by intensive selection, non-random mating, and mutation. Selection of Bali cattle in BPTU-HMT Bali province aimed to produce

Table 2. Polymorphisms of myostatin gene promoter in Bali cattle

\begin{tabular}{|c|c|c|c|c|c|c|c|c|}
\hline \multirow{2}{*}{ SNPs } & \multicolumn{3}{|c|}{ Genotype frequency } & \multicolumn{2}{|c|}{ Allele frequency } & \multirow{2}{*}{ Ho } & \multirow{2}{*}{$\mathrm{He}$} & \multirow{2}{*}{$x^{2}$} \\
\hline & $\mathrm{AA}$ & $\mathrm{AB}$ & $\mathrm{BB}$ & A & B & & & \\
\hline g. $-8350 \mathrm{C}>\mathrm{T}$ & 0.542 & 0.104 & 0.354 & 0.594 & 0.406 & 0.10 & 0.49 & ** \\
\hline g. $-8310 A>C$ & 0.542 & 0.042 & 0.416 & 0.563 & 0.437 & 0.04 & 0.51 & $* *$ \\
\hline g. $-8299 \mathrm{G}>\mathrm{A}$ & 0.042 & 0.062 & 0.896 & 0.927 & 0.073 & 0.06 & 0.14 & $* *$ \\
\hline g. $-8283 A>G$ & 0.042 & 0.104 & 0.854 & 0.094 & 0.906 & 0.10 & 0.17 & $* *$ \\
\hline g.8216G $>A$ & 0.875 & 0.083 & 0.042 & 0.083 & 0.917 & 0.08 & 0.15 & $* *$ \\
\hline g.- $-8205 A>G$ & 0.521 & 0.083 & 0.396 & 0.563 & 0.437 & 0.08 & 0.50 & $* *$ \\
\hline g. $-8168 A>G$ & 0.063 & 0.083 & 0.854 & 0.104 & 0.896 & 0.08 & 0.19 & $* *$ \\
\hline g. $-8109 \mathrm{~T}>\mathrm{G}$ & 0.542 & - & 0.458 & 0.542 & 0.458 & 0.00 & 0.50 & $* *$ \\
\hline g. $-8078 \mathrm{C}>\mathrm{T}$ & 0.729 & 0.229 & 0.042 & 0.844 & 0.156 & 0.23 & 0.27 & ns \\
\hline g. $-8077 \mathrm{G}>\mathrm{A}$ & 0.937 & 0.042 & 0.021 & 0.958 & 0.042 & 0.04 & 0.08 & $* *$ \\
\hline g. $-8029 \mathrm{~T}>\mathrm{C}$ & 0.479 & 0.208 & 0.313 & 0.583 & 0.417 & 0.21 & 0.49 & $* *$ \\
\hline g. $-8028 A>G$ & 0.354 & 0.292 & 0.354 & 0.500 & 0.500 & 0.29 & 0.51 & $* *$ \\
\hline g. $-8016 \mathrm{C}>\mathrm{T}$ & 0.666 & 0.188 & 0.146 & 0.760 & 0.240 & 0.19 & 0.37 & $* *$ \\
\hline g. $-7799 \mathrm{~T}>\mathrm{C}$ & 0.104 & 0.188 & 0.708 & 0.198 & 0.802 & 0.19 & 0.32 & $* *$ \\
\hline g. $-7996 \mathrm{G}>\mathrm{C}$ & 0.104 & 0.354 & 0.542 & 0.281 & 0.719 & 0.35 & 0.41 & ns \\
\hline g. $-7953 C>T$ & 0.063 & 0.104 & 0.833 & 0.115 & 0.885 & 0.10 & 0.21 & $* *$ \\
\hline g. $-7942 C>G$ & 0.916 & 0.042 & 0.042 & 0.938 & 0.062 & 0.04 & 0.12 & $* *$ \\
\hline g. $-7941 C>T$ & 0.708 & 0.084 & 0.208 & 0.750 & 0.250 & 0.08 & 0.38 & $* *$ \\
\hline g. $-7930 A>G$ & 0.792 & 0.188 & 0.020 & 0.885 & 0.115 & 0.19 & 0.21 & ns \\
\hline g. $-7905 \mathrm{~T}>\mathrm{C}$ & 0.542 & - & 0.458 & 0.542 & 0.458 & 0.00 & 0.50 & $* *$ \\
\hline
\end{tabular}

Note: $\mathrm{AA}=$ reference genotype; $\mathrm{AB}=$ heterozygote genotype; $\mathrm{BB}=$ mutant genotype; $\mathrm{A}=$ reference allele; $\mathrm{B}=$ mutant alelel; Ho= observed heterozygosis; $\mathrm{He}=$ expected heterozygosity; $\chi 2=$ Hardy-Weinberg equilibrium; (ns) not significant at $\alpha 5 \%(\mathrm{X} 2$ obs $\geq 3.84) ;\left({ }^{* *}\right)$ significant at $\alpha 1 \%(\mathrm{X} 2 \mathrm{obs} \geq 6.64)$; $\mathrm{n}=48$ heads.

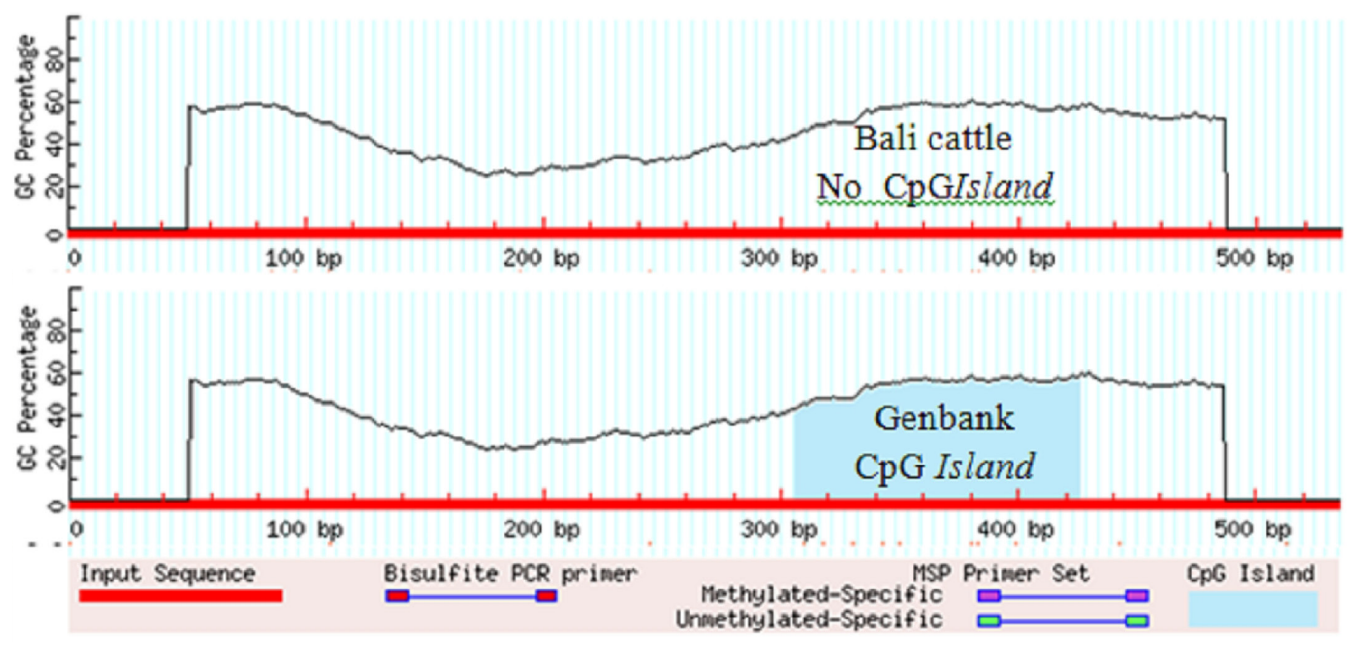

Figure 4. CpG island prediction in promoter myostatin gene in Bali cattle 
Table 3. Association of SNPs in MSTN promoter gene with growth traits in Bali cattle

\begin{tabular}{|c|c|c|c|c|c|c|c|c|c|}
\hline SNPs & Genotype & $\mathrm{n}$ & BW & WW & YW & ADG & SH & BL & CC \\
\hline \multirow[t]{3}{*}{ g. $-8350 C>T$} & $\mathrm{CC}$ & 26 & $17.65 \pm 1.15$ & $57.83 \pm 12.13$ & $89.83 \pm 21.25$ & $0.23 \pm 0.06$ & $92.08 \pm 1.14$ & $84.44 \pm 1.39$ & $109.80 \pm 1.91$ \\
\hline & $\mathrm{CT}$ & 5 & $17.00 \pm 0.00$ & $63.60 \pm 11.17$ & $100.60 \pm 19.89$ & $0.20 \pm 0.05$ & $93.54 \pm 2.41$ & $88.15 \pm 2.95$ & $112.31 \pm 4.05$ \\
\hline & TT & 17 & $16.92 \pm 1.73$ & $53.60 \pm 7.75$ & $81.47 \pm 14.40$ & $0.18 \pm 0.04$ & $90.97 \pm 1.44$ & $83.17 \pm 1.76$ & $106.24 \pm 2.41$ \\
\hline \multirow[t]{3}{*}{ g. $-8310 A>C$} & AA & 26 & $16.92 \pm 1.15$ & $57.83 \pm 12.13$ & $89.83 \pm 21.25$ & $0.20 \pm 0.06$ & $92.06 \pm 1.15$ & $84.42 \pm 1.42$ & $109.76 \pm 1.95$ \\
\hline & $\mathrm{AC}$ & 2 & $17.00 \pm 0.00$ & $57.00 \pm 15.68$ & $88.50 \pm 27.91$ & $0.20 \pm 0.08$ & $92.52 \pm 3.94$ & $87.38 \pm 4.87$ & $109.71 \pm 6.68$ \\
\hline & $\mathrm{CC}$ & 20 & $17.55 \pm 1.62$ & $56.00 \pm 9.35$ & $86.00 \pm 17.20$ & $0.19 \pm 0.05$ & $91.54 \pm 1.34$ & $84.10 \pm 1.65$ & $107.60 \pm 2.27$ \\
\hline \multirow[t]{3}{*}{ g. $-8299 \mathrm{G}>A$} & GG & 43 & $17.23 \pm 1.39$ & $56.13 \pm 9.71$ & $86.50 \pm 16.74$ & $0.19 \pm 0.05$ & $91.43 \pm 3.73$ & $83.81 \pm 1.05$ & $108.04 \pm 1.45$ \\
\hline & AG & 3 & $17.00 \pm 0.00$ & $63.33 \pm 15.68$ & $100.00 \pm 27.91$ & $0.23 \pm 0.08$ & $94.73 \pm 3.05$ & $90.37 \pm 3.75$ & $113.51 \pm 5.17$ \\
\hline & $\mathrm{AA}$ & 2 & $16.50 \pm 0.71$ & $64.50 \pm 10.58$ & $102.00 \pm 18.83$ & $0.23 \pm 0.04$ & $95.84 \pm 0.85$ & $87.21 \pm 4.58$ & $117.32 \pm 6.31$ \\
\hline \multirow[t]{3}{*}{ g. $-8283 A>G$} & $\mathrm{AA}$ & 2 & $16.50 \pm 0.71$ & $64.50 \pm 9.71$ & $102.00 \pm 16.74$ & $0.23 \pm 0.04$ & $95.82 \pm 3.68$ & $87.19 \pm 4.55$ & $117.28 \pm 6.21$ \\
\hline & AG & 5 & $17.80 \pm 0.50$ & $62.75 \pm 12.88$ & $98.75 \pm 22.94$ & $0.22 \pm 0.06$ & $95.44 \pm 2.63$ & $89.93 \pm 3.26$ & $114.95 \pm 4.45$ \\
\hline & GG & 41 & $17.28 \pm 1.42$ & $56.00 \pm 10.70$ & $86.27 \pm 19.04$ & $0.19 \pm 0.05$ & $91.26 \pm 0.85$ & $83.68 \pm 1.06$ & $107.73 \pm 1.44$ \\
\hline \multirow[t]{3}{*}{ g. $8216 \mathrm{G}>\mathrm{A}$} & GG & 2 & $17.24 \pm 0.71$ & $64.50 \pm 10.70$ & $102.00 \pm 19.04$ & $0.23 \pm 0.04$ & $95.82 \pm 3.68$ & $87.19 \pm 1.06$ & $117.28 \pm 6.21$ \\
\hline & AG & 4 & $17.00 \pm 0.00$ & $62.75 \pm 12.88$ & $98.75 \pm 22.94$ & $0.22 \pm 0.06$ & $95.44 \pm 2.63$ & $89.93 \pm 3.26$ & $114.95 \pm 4.45$ \\
\hline & AA & 42 & $16.50 \pm 1.41$ & $56.00 \pm 9.71$ & $86.27 \pm 16.74$ & $0.19 \pm 0.05$ & $91.26 \pm 0.85$ & $83.68 \pm 1.06$ & $107.73 \pm 1.44$ \\
\hline \multirow{3}{*}{ g. $-8205 A>G$} & AA & 25 & $17.92 \pm 1.18$ & $57.68 \pm 12.40$ & $89.59 \pm 21.72$ & $0.22 \pm 0.06$ & $91.82 \pm 1.14$ & $84.23 \pm 1.40$ & $109.33 \pm 1.93$ \\
\hline & AG & 4 & $17.00 \pm 0.00$ & $62.75 \pm 12.88$ & $98.75 \pm 22.94$ & $0.20 \pm 0.06$ & $95.46 \pm 2.68$ & $89.95 \pm 3.28$ & $115.01 \pm 4.53$ \\
\hline & GG & 19 & $17.58 \pm 1.62$ & $54.82 \pm 8.10$ & $83.82 \pm 15.01$ & $0.18 \pm 0.04$ & $91.07 \pm 1.32$ & $83.37 \pm 1.61$ & $106.76 \pm 2.23$ \\
\hline \multirow[t]{3}{*}{ g. $-8168 A>G$} & $\mathrm{AA}$ & 3 & $16.33 \pm 0.58$ & $64.50 \pm 9.71$ & $102.00 \pm 16.74$ & $0.23 \pm 0.04$ & $95.82 \pm 3.68$ & $87.19 \pm 4.55$ & $117.28 \pm 6.21$ \\
\hline & AG & 4 & $17.00 \pm 0.00$ & $62.75 \pm 12.88$ & $98.75 \pm 22.94$ & $0.22 \pm 0.06$ & $95.44 \pm 2.63$ & $89.93 \pm 3.26$ & $114.95 \pm 4.45$ \\
\hline & GG & 41 & $17.27 \pm 1.42$ & $56.00 \pm 10.70$ & $86.27 \pm 19.04$ & $0.19 \pm 0.05$ & $91.26 \pm 0.85$ & $83.68 \pm 1.06$ & $107.73 \pm 1.44$ \\
\hline \multirow[t]{2}{*}{ g. $-8109 \mathrm{~T}>\mathrm{G}$} & TT & 26 & $17.35 \pm 1.45$ & $57.04 \pm 9.45$ & $88.00 \pm 22.10$ & $0.19 \pm 0.05$ & $92.30 \pm 1.13$ & $84.91 \pm 1.40$ & $109.23 \pm 1.93$ \\
\hline & GG & 22 & $17.00 \pm 1.22$ & $57.00 \pm 12.63$ & $88.35 \pm 17.31$ & $0.19 \pm 0.06$ & $91.37 \pm 1.21$ & $83.88 \pm 1.50$ & $108.44 \pm 2.07$ \\
\hline \multirow[t]{3}{*}{ g. $-8078 \mathrm{C}>\mathrm{T}$} & $\mathrm{CC}$ & 35 & $18.50 \pm 1.39$ & $57.58 \pm 10.82$ & $89.10 \pm 19.44$ & $0.20 \pm 0.05$ & $92.33 \pm 0.96$ & $84.60 \pm 1.20$ & $109.50 \pm 1.60$ \\
\hline & $\mathrm{CT}$ & 11 & $17.23 \pm 0.92$ & $54.20 \pm 10.86$ & $83.40 \pm 19.29$ & $0.18 \pm 0.05$ & $90.32 \pm 1.70$ & $83.55 \pm 2.13$ & $105.41 \pm 2.83$ \\
\hline & TT & 2 & $16.82 \pm 2.12$ & $62.50 \pm 16.31$ & $97.50 \pm 27.39$ & $0.22 \pm 0.07$ & $92.53 \pm 3.87$ & $86.17 \pm 4.85$ & $116.46 \pm 6.44$ \\
\hline \multirow[t]{3}{*}{ g. $-8077 \mathrm{G}>\mathrm{A}$} & GG & 45 & $17.22 \pm 1.38$ & $57.00 \pm 0.00$ & $88.10 \pm 0.00$ & $0.19 \pm 0.05$ & $91.66 \pm 0.84$ & $84.19 \pm 1.06$ & $108.65 \pm 1.46$ \\
\hline & AG & 2 & $17.00 \pm 0.00$ & $57.00 \pm 15.68$ & $88.50 \pm 27.91$ & $0.20 \pm 0.08$ & $92.58 \pm 3.86$ & $87.41 \pm 4.84$ & $109.51 \pm 6.66$ \\
\hline & $\mathrm{AA}$ & 1 & $16.00 \pm 0.00$ & $58.00 \pm 11.04$ & $90.00 \pm 19.67$ & $0.20 \pm 0.00$ & $98.31 \pm 5.41$ & $88.01 \pm 6.78$ & $115.54 \pm 9.34$ \\
\hline \multirow[t]{3}{*}{ g. $-8029 \mathrm{~T}>\mathrm{C}$} & $\mathrm{CC}$ & 15 & $17.60 \pm 1.76$ & $55.67 \pm 10.52$ & $85.47 \pm 18.71$ & $0.19 \pm 0.05$ & $90.72 \pm 1.40$ & $83.69 \pm 1.77$ & $106.72 \pm 2.40$ \\
\hline & $\mathrm{CT}$ & 10 & $17.00 \pm 1.33$ & $59.50 \pm 11.65$ & $93.00 \pm 20.33$ & $0.21 \pm 0.05$ & $91.39 \pm 1.70$ & $84.32 \pm 2.15$ & $108.95 \pm 2.91$ \\
\hline & TT & 23 & $17.00 \pm 0.90$ & $56.78 \pm 11.20$ & $87.72 \pm 20.15$ & $0.19 \pm 0.06$ & $93.07 \pm 1.30$ & $85.10 \pm 1.63$ & $110.56 \pm 2.22$ \\
\hline \multirow[t]{3}{*}{ g. $-8028 \mathrm{~A}>\mathrm{G}$} & AA & 17 & $17.47 \pm 1.70$ & $56.82 \pm 10.68$ & $87.59 \pm 19.06$ & $0.19 \pm 0.05$ & $91.65 \pm 1.32$ & $84.53 \pm 1.63$ & $108.62 \pm 2.26$ \\
\hline & AG & 17 & $17.12 \pm 1.14$ & $59.77 \pm 11.75$ & $93.62 \pm 20.64$ & $0.21 \pm 0.06$ & $91.87 \pm 1.51$ & $85.25 \pm 1.87$ & $108.90 \pm 2.58$ \\
\hline & GG & 14 & $16.93 \pm 0.99$ & $54.54 \pm 10.53$ & $83.46 \pm 18.94$ & $0.18 \pm 0.05$ & $92.13 \pm 1.54$ & $83.50 \pm 1.91$ & $109.12 \pm 2.63$ \\
\hline \multirow[t]{3}{*}{ g. $-8016 \mathrm{C}>\mathrm{T}$} & $\mathrm{CC}$ & 32 & $17.20 \pm 1.33$ & $56.93 \pm 10.06$ & $87.93 \pm 18.36$ & $0.19 \pm 0.05$ & $92.46 \pm 1.01$ & $84.52 \pm 1.27$ & $109.12 \pm 1.75$ \\
\hline & $\mathrm{CT}$ & 9 & $17.22 \pm 1.20$ & $58.88 \pm 12.71$ & $91.75 \pm 22.20$ & $0.20 \pm 0.06$ & $91.26 \pm 1.90$ & $84.47 \pm 2.39$ & $108.19 \pm 3.28$ \\
\hline & $\mathrm{TT}$ & 7 & $17.14 \pm 1.57$ & $55.29 \pm 13.40$ & $85.00 \pm 22.93$ & $0.19 \pm 0.06$ & $90.17 \pm 2.02$ & $84.05 \pm 2.55$ & $108.58 \pm 3.50$ \\
\hline \multirow[t]{3}{*}{ g. $-7799 \mathrm{~T}>\mathrm{C}$} & $\mathrm{CC}$ & 34 & $17.35 \pm 1.52$ & $55.81 \pm 10.68$ & $85.77 \pm 18.95$ & $0.19 \pm 0.05$ & $91.36 \pm 0.97$ & $83.39 \pm 1.18$ & $107.88 \pm 1.65$ \\
\hline & $\mathrm{CT}$ & 9 & $16.89 \pm 0.33$ & $58.88 \pm 10.67$ & $92.00 \pm 19.18$ & $0.21 \pm 0.05$ & $92.22 \pm 2.02$ & $86.13 \pm 2.46$ & $109.94 \pm 3.44$ \\
\hline & TT & 5 & $16.60 \pm 0.55$ & $62.75 \pm 13.71$ & $99.00 \pm 24.31$ & $0.23 \pm 0.07$ & $95.04 \pm 2.66$ & $88.99 \pm 3.24$ & $114.23 \pm 4.52$ \\
\hline \multirow[t]{3}{*}{ g. $-7996 \mathrm{G}>\mathrm{C}$} & $\mathrm{CC}$ & 26 & $17.24 \pm 1.47$ & $55.52 \pm 9.31$ & $85.17 \pm 17.09$ & $0.19 \pm 0.05$ & $91.45 \pm 1.10$ & $83.68 \pm 1.40$ & $107.41 \pm 1.87$ \\
\hline & CG & 17 & $16.88 \pm 0.99$ & $57.27 \pm 13.23$ & $89.07 \pm 23.15$ & $0.20 \pm 0.06$ & $91.23 \pm 1.38$ & $84.99 \pm 1.76$ & $109.05 \pm 2.36$ \\
\hline & GG & 5 & $17.40 \pm 1.52$ & $63.20 \pm 9.92$ & $99.20 \pm 16.93$ & $0.22 \pm 0.04$ & $95.75 \pm 2.39$ & $86.21 \pm 3.05$ & $115.01 \pm 4.07$ \\
\hline g. $-7953 C>T$ & $\mathrm{CC}$ & 3 & $17.25 \pm 0.58$ & $64.50 \pm 9.71$ & $102.00 \pm 16.74$ & $0.23 \pm 0.04$ & $95.84 \pm 3.76$ & $87.20 \pm 4.59$ & $117.30 \pm 6.30$ \\
\hline & $\mathrm{CT}$ & 5 & $17.20 \pm 0.45$ & $59.40 \pm 13.59$ & $92.40 \pm 24.39$ & $0.21 \pm 0.07$ & $93.27 \pm 2.43$ & $88.88 \pm 2.96$ & $112.58 \pm 4.07$ \\
\hline & TT & 40 & $16.33 \pm 1.41$ & $56.28 \pm 10.70$ & $86.81 \pm 19.03$ & $0.19 \pm 0.05$ & $91.44 \pm 0.89$ & $83.65 \pm 1.08$ & $107.86 \pm 1.49$ \\
\hline g. $-7942 C>G$ & $\mathrm{CC}$ & 44 & $17.23 \pm 1.36$ & $56.10 \pm 10.59$ & $86.48 \pm 18.85$ & $0.19 \pm 0.05$ & $91.50 \pm 0.82$ & $83.98 \pm 1.02$ & $108.03 \pm 1.37$ \\
\hline & CG & 2 & $17.00 \pm 0.00$ & $68.50 \pm 11.11$ & $109.00 \pm 19.78$ & $0.25 \pm 0.05$ & $98.45 \pm 3.74$ & $92.57 \pm 4.65$ & $120.29 \pm 6.24$ \\
\hline & GG & 2 & $16.50 \pm 0.71$ & $71.00 \pm 0.00$ & $114.00 \pm 0.00$ & $0.27 \pm 0.00$ & $93.31 \pm 5.39$ & $86.33 \pm 6.70$ & $119.36 \pm 9.00$ \\
\hline g. $-7941 C>T$ & $\mathrm{CC}$ & 34 & $17.18 \pm 1.40$ & $57.58 \pm 11.29$ & $89.23 \pm 20.09$ & $0.20 \pm 0.06$ & $91.84 \pm 0.99$ & $84.17 \pm 1.22$ & $108.53 \pm 1.68$ \\
\hline & $\mathrm{CT}$ & 4 & $16.50 \pm 1.00$ & $59.75 \pm 12.60$ & $93.75 \pm 22.29$ & $0.21 \pm 0.06$ & $93.73 \pm 2.70$ & $87.20 \pm 3.34$ & $112.16 \pm 4.61$ \\
\hline & TT & 10 & $17.50 \pm 1.08$ & $53.50 \pm 8.88$ & $81.25 \pm 15.84$ & $0.17 \pm 0.04$ & $91.05 \pm 2.00$ & $84.04 \pm 2.48$ & $108.49 \pm 3.42$ \\
\hline g. $-7930 A>G$ & AA & 38 & $17.24 \pm 1.44$ & $57.71 \pm 10.84$ & $89.32 \pm 19.29$ & $0.20 \pm 0.05$ & $92.32 \pm 0.92$ & $84.70 \pm 1.12$ & $109.75 \pm 1.54$ \\
\hline & AG & 9 & $17.00 \pm 0.71$ & $56.13 \pm 10.96$ & $86.75 \pm 19.73$ & $0.19 \pm 0.05$ & $90.41 \pm 1.88$ & $84.71 \pm 2.29$ & $106.59 \pm 3.17$ \\
\hline & GG & 1 & $17.00 \pm 0.00$ & $41.00 \pm 0.00$ & $60.00 \pm 0.00$ & $0.12 \pm 0.00$ & $88.26 \pm 5.46$ & $73.42 \pm 6.65$ & $97.08 \pm 9.17$ \\
\hline g. $-7905 \mathrm{~T}>\mathrm{C}$ & $\mathrm{CC}$ & 22 & $17.00 \pm 1.20$ & $57.04 \pm 12.63$ & $88.35 \pm 22.10$ & $0.19 \pm 0.06$ & $91.37 \pm 1.21$ & $83.88 \pm 1.50$ & $108.44 \pm 2.07$ \\
\hline & TT & 26 & $17.35 \pm 1.41$ & $57.04 \pm 9.45$ & $88.00 \pm 17.31$ & $0.19 \pm 0.05$ & $92.30 \pm 1.13$ & $84.91 \pm 1.40$ & $109.23 \pm 1.93$ \\
\hline
\end{tabular}

Note: $\mathrm{BW}=$ birth weight; $\mathrm{WW}=$ weaning weight $(205 \mathrm{~d})$; $\mathrm{YW}=$ yealing weight $(365 \mathrm{~d})$; $\mathrm{SH}=$ shoulder height; $\mathrm{BL}=$ Body length; $\mathrm{CC}=$ chest circumstance. 
Table 4. Association of SNPs in MSTN with muscling traits in Bali cattle

\begin{tabular}{|c|c|c|c|c|c|c|c|c|}
\hline SNPs & Genotype & $\mathrm{n}$ & LDT & BFT & RT & RFT & MS & PIMF \\
\hline \multirow[t]{3}{*}{ g. $-8350 C>T$} & CC & 14 & $30.17 \pm 4.88$ & $1.40 \pm 0.33$ & $37.61 \pm 5.06$ & $1.09 \pm 0.27$ & $2.24 \pm 0.85$ & $3.65 \pm 1.53$ \\
\hline & $\mathrm{CT}$ & 5 & $32.44 \pm 2.71$ & $1.22 \pm 0.20$ & $36.74 \pm 4.80$ & $0.91 \pm 0.28$ & $2.57 \pm 0.59$ & $4.13 \pm 1.85$ \\
\hline & $\mathrm{TT}$ & 12 & $28.86 \pm 5.47$ & $1.24 \pm 0.23$ & $34.95 \pm 4.56$ & $0.89 \pm 0.29$ & $1.72 \pm 0.93$ & $2.65 \pm 1.54$ \\
\hline \multirow[t]{3}{*}{ g. $-8310 A>C$} & AA & 14 & $30.17 \pm 4.88$ & $1.40 \pm 0.33$ & $37.61 \pm 5.06$ & $1.09 \pm 0.27$ & $2.24 \pm 0.85$ & $3.65 \pm 1.53$ \\
\hline & $\mathrm{CA}$ & 2 & $30.26 \pm 1.77$ & $1.34 \pm 0.06$ & $34.01 \pm 0.89$ & $0.74 \pm 0.28$ & $2.68 \pm 1.02$ & $4.54 \pm 3.07$ \\
\hline & $\mathrm{CC}$ & 15 & $29.87 \pm 5.35$ & $1.22 \pm 0.22$ & $35.67 \pm 4.83$ & $0.92 \pm 0.28$ & $1.89 \pm 0.89$ & $2.91 \pm 1.54$ \\
\hline \multirow[t]{3}{*}{ g. $-8299 \mathrm{G}>\mathrm{A}$} & $\mathrm{AA}^{*}$ & 1 & $35.11 \pm 0.00$ & $2.41 \pm 0.00$ & $45.94 \pm 0.00$ & $1.02 \pm 0.00$ & $4.00 \pm 0.00$ & $7.24 \pm 0.00$ \\
\hline & AG & 3 & $32.27 \pm 3.70$ & $1.22 \pm 0.21$ & $33.87 \pm 0.68$ & $0.74 \pm 0.20$ & $2.61 \pm 0.73$ & $4.54 \pm 2.17$ \\
\hline & GG & 27 & $29.77 \pm 4.98$ & $1.32 \pm 0.30$ & $36.68 \pm 5.01$ & $1.01 \pm 0.27$ & $2.04 \pm 0.87$ & $3.22 \pm 1.51$ \\
\hline \multirow[t]{3}{*}{ g. $-8283 A>G$} & $\mathrm{AA}^{*}$ & 1 & $35.12 \pm 0.00$ & $2.41 \pm 0.00$ & $45.94 \pm 0.00$ & $1.02 \pm 0.00$ & $4.00 \pm 0.00$ & $7.24 \pm 0.00$ \\
\hline & AG & 4 & $31.40 \pm 3.49$ & $1.28 \pm 0.21$ & $34.29 \pm 1.00$ & $0.76 \pm 0.17$ & $2.48 \pm 0.65$ & $4.20 \pm 1.90$ \\
\hline & GG & 26 & $29.81 \pm 5.08$ & $1.31 \pm 0.31$ & $36.73 \pm 5.10$ & $1.02 \pm 0.28$ & $2.04 \pm 0.88$ & $3.22 \pm 1.54$ \\
\hline \multirow[t]{3}{*}{ g.8216G $>A$} & AA & 26 & $31.40 \pm 5.08$ & $1.31 \pm 0.31$ & $36.73 \pm 5.10$ & $1.02 \pm 0.28$ & $2.04 \pm 0.88$ & $3.22 \pm 1.54$ \\
\hline & AG & 4 & $29.81 \pm 3.49$ & $1.28 \pm 0.21$ & $34.29 \pm 1.00$ & $0.76 \pm 0.17$ & $2.48 \pm 0.65$ & $4.20 \pm 1.90$ \\
\hline & $\mathrm{GG}^{*}$ & 1 & $35.11 \pm 0.00$ & $2.41 \pm 0.00$ & $45.94 \pm 0.00$ & $1.02 \pm 0.00$ & $4.00 \pm 0.00$ & $7.24 \pm 0.00$ \\
\hline \multirow[t]{3}{*}{ g. $-8205 A>G$} & AA & 13 & $30.28 \pm 5.08$ & $1.40 \pm 0.34$ & $37.79 \pm 5.24$ & $1.12 \pm 0.27$ & $2.25 \pm 0.88$ & $3.69 \pm 1.61$ \\
\hline & AG & 4 & $31.40 \pm 3.49$ & $1.28 \pm 0.21$ & $34.29 \pm 1.00$ & $0.76 \pm 0.17$ & $2.48 \pm 0.65$ & $4.20 \pm 1.90$ \\
\hline & GG & 14 & $29.41 \pm 5.23$ & $1.28 \pm 0.23$ & $35.82 \pm 4.98$ & $0.93 \pm 0.29$ & $1.84 \pm 0.91$ & $2.78 \pm 1.53$ \\
\hline \multirow[t]{3}{*}{ g. $-8168 A>G$} & $\mathrm{AA}^{*}$ & 1 & $35.11 \pm 0.00$ & $2.41 \pm 0.00$ & $45.94 \pm 0.00$ & $1.02 \pm 0.00$ & $4.00 \pm 0.00$ & $7.24 \pm 0.00$ \\
\hline & AG & 26 & $29.81 \pm 3.49$ & $1.28 \pm 0.21$ & $34.29 \pm 1.00$ & $0.76 \pm 0.17$ & $2.48 \pm 0.65$ & $4.20 \pm 1.90$ \\
\hline & GG & 4 & $31.40 \pm 5.08$ & $1.31 \pm 0.31$ & $36.73 \pm 5.10$ & $1.02 \pm 0.28$ & $2.04 \pm 0.88$ & $3.22 \pm 1.54$ \\
\hline \multirow[t]{2}{*}{ g. $-8109 \mathrm{~T}>\mathrm{G}$} & GG & 12 & $30.28 \pm 5.08$ & $1.40 \pm 0.34$ & $37.79 \pm 5.24$ & $1.12 \pm 0.27$ & $2.25 \pm 0.88$ & $3.69 \pm 1.61$ \\
\hline & $\mathrm{TT}$ & 19 & $29.85 \pm 4.88$ & $1.25 \pm 0.22$ & $35.48 \pm 4.42$ & $0.89 \pm 0.27$ & $1.99 \pm 0.88$ & $3.12 \pm 1.67$ \\
\hline \multirow[t]{3}{*}{ g. $-8078 \mathrm{C}>\mathrm{T}$} & $\mathrm{CC}$ & 24 & $30.32 \pm 4.90$ & $1.29 \pm 0.26$ & $36.47 \pm 4.91$ & $1.00 \pm 0.26$ & $1.94 \pm 0.89$ & $3.13 \pm 1.69$ \\
\hline & $\mathrm{CT}$ & 4 & $29.19 \pm 6.45$ & $1.28 \pm 0.39$ & $35.99 \pm 6.13$ & $0.88 \pm 0.41$ & $2.14 \pm 0.55$ & $3.08 \pm 1.57$ \\
\hline & $\mathrm{TT}$ & 2 & $28.12 \pm 0.20$ & $1.51 \pm 0.49$ & $36.34 \pm 2.30$ & $1.02 \pm 0.24$ & $3.89 \pm 0.00^{*}$ & $6.48 \pm 0.00^{*}$ \\
\hline \multirow[t]{3}{*}{ g.-8077G $>A$} & $\mathrm{AA}^{*}$ & 1 & $35.11 \pm 0.00$ & $2.41 \pm 0.00$ & $45.94 \pm 0.00$ & $1.02 \pm 0.00$ & $4.00 \pm 0.00$ & $7.24 \pm 0.00$ \\
\hline & AG & 2 & $30.26 \pm 1.77$ & $1.34 \pm 0.06$ & $34.01 \pm 0.89$ & $0.74 \pm 0.28$ & $2.68 \pm 1.02$ & $4.54 \pm 3.07$ \\
\hline & GG & 28 & $30.01 \pm 5.04$ & $1.30 \pm 0.30$ & $36.57 \pm 4.95$ & $1.00 \pm 0.27$ & $2.06 \pm 0.86$ & $3.27 \pm 1.51$ \\
\hline \multirow[t]{3}{*}{ g. $-8029 \mathrm{~T}>\mathrm{C}$} & $\mathrm{CC}$ & 12 & $29.16 \pm 5.19$ & $1.27 \pm 0.37$ & $36.59 \pm 5.99$ & $1.00 \pm 0.26$ & $2.06 \pm 0.77$ & $3.62 \pm 1.50$ \\
\hline & $\mathrm{CT}$ & 5 & $32.53 \pm 5.35$ & $1.40 \pm 0.25$ & $39.50 \pm 4.73$ & $0.88 \pm 0.32$ & $2.90 \pm 0.26$ & $3.33 \pm 0.91$ \\
\hline & TT & 14 & $29.85 \pm 4.46$ & $1.31 \pm 0.22$ & $35.03 \pm 3.11$ & $1.01 \pm 0.28$ & $1.88 \pm 1.07$ & $3.12 \pm 1.95$ \\
\hline \multirow[t]{3}{*}{ g. $-8028 A>G$} & AA & 13 & $29.14 \pm 5.17$ & $1.27 \pm 0.37$ & $35.74 \pm 5.40$ & $0.94 \pm 0.28$ & $2.05 \pm 0.77$ & $3.42 \pm 1.40$ \\
\hline & AG & 8 & $32.87 \pm 4.55$ & $1.32 \pm 0.24$ & $38.88 \pm 4.99$ & $0.98 \pm 0.24$ & $2.64 \pm 0.83$ & $4.15 \pm 1.49$ \\
\hline & GG & 10 & $28.81 \pm 4.24$ & $1.34 \pm 0.24$ & $35.21 \pm 3.49$ & $1.03 \pm 0.32$ & $1.78 \pm 1.04$ & $2.72 \pm 1.76$ \\
\hline \multirow[t]{3}{*}{ g. $-8016 C>T$} & $\mathrm{CC}$ & 23 & $30.17 \pm 4.84$ & $1.26 \pm 0.21$ & $35.67 \pm 4.25$ & $0.97 \pm 0.26$ & $1.87 \pm 0.89$ & $2.99 \pm 1.65$ \\
\hline & $\mathrm{CT}$ & 3 & $30.87 \pm 5.90$ & $1.40 \pm 0.35$ & $40.97 \pm 5.43$ & $0.90 \pm 0.34$ & $3.22 \pm 0.26$ & $3.65 \pm 0.80$ \\
\hline & $\mathrm{TT}$ & 5 & $28.87 \pm 5.46$ & $1.44 \pm 0.50$ & $36.89 \pm 6.29$ & $1.07 \pm 0.34$ & $2.39 \pm 1.11$ & $4.69 \pm 2.13$ \\
\hline \multirow[t]{3}{*}{ g. $-7799 \mathrm{~T}>\mathrm{C}$} & $\mathrm{CC}$ & 24 & $29.64 \pm 5.03$ & $1.31 \pm 0.32$ & $36.19 \pm 4.92$ & $0.96 \pm 0.28$ & $2.08 \pm 0.91$ & $3.31 \pm 1.57^{\mathrm{ab}}$ \\
\hline & $\mathrm{CT}$ & 4 & $31.03 \pm 4.48$ & $1.36 \pm 0.10$ & $39.13 \pm 4.79$ & $1.22 \pm 0.36$ & $1.78 \pm 0.54$ & $2.48 \pm 0.84^{\mathrm{b}}$ \\
\hline & $\mathrm{TT}$ & 3 & $32.65 \pm 5.15$ & $1.14 \pm 0.23$ & $33.49 \pm 0.16$ & $0.84 \pm 0.15$ & $2.94 \pm 0.65$ & $5.62 \pm 1.54^{\mathrm{a}}$ \\
\hline \multirow[t]{3}{*}{ g.-7996G>C } & $\mathrm{CC}$ & 18 & $30.10 \pm 5.13$ & $1.29 \pm 0.26$ & $36.24 \pm 4.81$ & $0.96 \pm 0.30$ & $1.92 \pm 0.87$ & $2.86 \pm 1.48$ \\
\hline & GC & 9 & $30.11 \pm 4.03$ & $1.32 \pm 0.26$ & $36.55 \pm 4.08$ & $1.04 \pm 0.25$ & $2.42 \pm 0.84$ & $4.13 \pm 1.84$ \\
\hline & GG & 4 & $29.34 \pm 7.41$ & $1.37 \pm 0.59$ & $36.93 \pm 8.53$ & $0.94 \pm 0.25$ & $2.16 \pm 1.21$ & $3.81 \pm 2.07$ \\
\hline g. $-7953 \mathrm{C}>\mathrm{T}$ & $\mathrm{CC}^{*}$ & 1 & $35.11 \pm 0.00$ & $2.41 \pm 0.00$ & $45.94 \pm 0.00$ & $1.02 \pm 0.00$ & $4.00 \pm 0.00$ & $7.24 \pm 0.00$ \\
\hline & $\mathrm{CT}$ & 5 & $31.18 \pm 3.06$ & $1.30 \pm 0.19$ & $34.94 \pm 1.70$ & $0.91 \pm 0.35$ & $2.34 \pm 0.64$ & $4.22 \pm 1.64$ \\
\hline & $\mathrm{TT}$ & 25 & $29.79 \pm 5.18$ & $1.31 \pm 0.31$ & $36.69 \pm 5.20$ & $1.00 \pm 0.26$ & $2.05 \pm 0.90$ & $3.17 \pm 1.55$ \\
\hline g. $-7942 C>G$ & $\mathrm{CC}$ & 29 & $29.84 \pm 4.90$ & $1.31 \pm 0.29$ & $36.53 \pm 4.96$ & $1.00 \pm 0.28$ & $2.08 \pm 0.91$ & $3.32 \pm 1.66$ \\
\hline & GC & 2 & $32.54 \pm 5.31$ & $1.22 \pm 0.34$ & $34.57 \pm 1.37$ & $0.78 \pm 0.08$ & $2.29 \pm 0.26$ & $3.85 \pm 0.96$ \\
\hline & GG & 0 & & & & & & --------- \\
\hline g. $-7941 \mathrm{C}>\mathrm{T}$ & $\mathrm{CC}$ & 21 & $30.03 \pm 4.95$ & $1.31 \pm 0.31$ & $37.14 \pm 5.40$ & $1.03 \pm 0.26$ & $2.09 \pm 0.74$ & $2.99 \pm 1.57^{b}$ \\
\hline & $\mathrm{CT}$ & 4 & $33.15 \pm 5.00$ & $1.29 \pm 0.22$ & $35.62 \pm 3.05$ & $0.91 \pm 0.17$ & $2.66 \pm 0.66$ & $4.81 \pm 0.84^{\mathrm{a}}$ \\
\hline & $\mathrm{TT}$ & 6 & $27.93 \pm 4.10$ & $1.31 \pm 0.26$ & $34.47 \pm 3.36$ & $0.88 \pm 0.39$ & $1.86 \pm 1.24$ & $2.71 \pm 1.54^{\mathrm{b}}$ \\
\hline g. $-7930 A>G$ & AA & 27 & $30.11 \pm 4.77$ & $1.30 \pm 0.28$ & $36.45 \pm 4.74$ & $0.98 \pm 0.26$ & $2.09 \pm 0.89$ & $3.39 \pm 1.66$ \\
\hline & $\mathrm{AG}$ & 3 & $30.15 \pm 7.55$ & $1.36 \pm 0.44$ & $37.03 \pm 7.06$ & $0.94 \pm 0.47$ & $2.57 \pm 0.67$ & $3.62 \pm 1.40$ \\
\hline & $\mathrm{GG}^{*}$ & 1 & $28.20 \pm 0.00$ & $1.46 \pm 0.00$ & $34.26 \pm 0.00$ & $1.11 \pm 0.00$ & $1.00 \pm 0.00$ & $1.63 \pm 0.00$ \\
\hline g. $-7905 T>C$ & $\mathrm{CC}$ & 12 & $30.28 \pm 5.08$ & $1.40 \pm 0.34$ & $37.79 \pm 5.24$ & $1.12 \pm 0.27$ & $2.25 \pm 0.88$ & $3.69 \pm 1.61$ \\
\hline & $\mathrm{TT}$ & 19 & $29.85 \pm 4.88$ & $1.25 \pm 0.22$ & $35.48 \pm 4.42$ & $0.89 \pm 0.27$ & $1.99 \pm 0.88$ & $3.12 \pm 1.67$ \\
\hline
\end{tabular}

Note: Means in the same column with different superscripts differ significantly $(\mathrm{P}<0.05)$; LTD= Longissimus dorsi thickness; $\mathrm{BFT}=\mathrm{Back}$ fat thickness; $\mathrm{RT}=$ Rump thickness; RFT= Rump fat thickness; MS= Marbing score; PIMF= Intramuscular fat percentage. 
superior bull. The mutation in Bali cattle is likely due to the efforts of these cattle to adapt harsh environmental conditions. The Ho value lower than $\mathrm{He}$ indicated that an inbreeding probability were occured in this population (Nassiry et al., 2009). Polymorphism in promoter region was also identified by $\mathrm{He}$ et al. (2013) at locus -371 of MSTN promoter gene which has 18 polymorphic SNPs in Qinchuan cattle. The most valuable meat is from longissimus dorsi which is in this research overall average of LDT and BFT were $33.047 \pm 5.077 \mathrm{~mm}$ and $1.455 \pm 0.348 \mathrm{~mm}$, respectively. Putri et al. (2015) showed that LTD and BFT in adult Bali cattle (more than 3 years) was $57.577 \mathrm{~mm}$ to $63.818 \mathrm{~mm}$ and $1.935 \mathrm{~mm}$ to 2.324 $\mathrm{mm}$, respectively.

\section{Association of Single Nucleotide Polymorphism (SNPs) in Myostatin Promoter Region with Growth and Muscling Traits in Bali Cattle}

The study revealed that there were no SNPs in promoter region of MSTN gene in Bali cattle had significant association with growth traits $(\mathrm{P}<0.05)$. Zhang et al. (2007) also found no significant association between MSTN promoter with birth weight, body weight at 6 , 12, 18, and 24 mo. The lack of association between the SNPs in MSTN gene and growth traits was also found in Hanwoo cattle (Han et al., 2012). Furthermore, association of MSTN promoter gene showed no significant effect on morphological measurement as performed by Sarti et al. (2014).

Two SNPs in MSTN promoter region (g.-7799T>C and g.-7941C $>\mathrm{T}$ ) showed significant association with PIMF (P<0.05) (Table 4). Han et al. (2012) found association between MSTN promoter gene with meat quality index and fat colour index in Hanwoo cattle. The genotype of AA and AT in Hanwoo cattle had higher meat quality index and fat colour index than AA genotype. The expression of MSTN gene is inversely related to the other myogenic expression (Shibata et al., 2006). However, MSTN expression did not disturbed other myogenic expressions, such as Myog, Myf5, and MyoD and increased muscle mass. Futhermore, this mutation was able to reduce adiposity both of white fat and brown fat affected by neighboring muscle fiber (Li et al., 2015). Myostatin significantly inhibited differentiation of preadipocyte by cytokine from muscle fiber (Li et al., 2015). Moreover, they play cross role in muscle-fat which might regulate fat ratio in muscle such as IMF percentage (Sun et al., 2016). Promoter sequence was analysed in mammalian like cattle, pig, sheep, goat, human and mice. The mutation in TATA, CACCC, and AT1 has significantly decreased promoter activity, although mutation in AT2 and PAL likely to increase promoter activity (Allen \& Du, 2008).

\section{CONCLUSION}

It could be concluded that myostatin (MSTN) promoter gene was polymorphic in Bali cattle and there were 2 SNPs (g.-7799T>C and g.-7941C>T) associated with carcass quality.

\section{ACKNOWLEDGEMENT}

This research was financially supported by Ministry of Research Technology and Higher Education (12/SEK/INSINAS/PPK/IV/2015) and Indonesia Endowment Fund for Education. Authors also greatly acknowledged the Bali cattle Breeding Centre (BPTUHMT) Bali Province for providing growth trait data and blood sample of Bali cattle that used in this study.

\section{REFERENCES}

Allen, D. L. \& M. Du. 2008. Comparative functional analysis of the cow and mouse myostatin genes reveals novel regulatory elements in their upstream promoter regions. Compar. Biochem. Physi. 150:432-439. http://dx.doi. org/10.1016/j.cbpb.2008.05.002

Allendrof, F. W., G. Luikart, \& S. N. Aitken. 2013. Conservation and the genetics of populations. $2^{\text {nd }} E d$. Wiley-Blackwell Publishing, Chicester, UK.

[BSN] National Standarization Agency of Indonesia (in Indonesian: Badan Standarisasi Nasional). 2015. Bibit Sapi potong. Bagian 4: Bali. SNI 7651.4:2015. Badan Standarisasi Nasional, Jakarta.

Carninci, P., A. Sandelin, B. Lenhard, S. Katayama, K. Shimokawa, J. Ponjavic, C. A. Semple, M. S. Taylor, P. G. Engstrom, \& M. C. Frith. 2006. Genome-wide analysis of mammalian promoter architecture and evolution. Nat. Genet. 38:626-635. http://dx.doi.org/10.1038/ng1789

Deaton, A. M. \& A. P. Bird. 2011. CpG island and the regulation of transcription. Genes Dev. 25:1010-1022.

Deaton, A. V., D. Wilson, \& G. Rouse. 2000. USOFT: An ultrasound image analysis software for beef quality research. Beef research report. A.S. Leaflet R1437. Iowa University. Iowa. http://dx.doi.org/10.1101/gad.2037511

Doherty, R., C. O' Farrelly, \& K. G. Meade. 2014. Comparative epigenetics: relevance to the regulation of production and health traits in cattle. Anim Genet. 45:3-14. http://dx.doi. org/10.1111/age.12140

Elliott B., D. Renshaw, S. Getting \& R. Mackenzie. 2012. The central role of myostatin in skeletal muscle and whole body homesostasis. Acta Physiologica 205:324-340. http:// dx.doi.org/10.1111/j.1748-1716.2012.02423.x

Gill, J. L., S. C. Bishop, C. Mc Corquodale, J. L. Williams, \& P. Wiener. 2008. Associations between the 11-bp deletion in the myostatin gene and carcass quality in Angus-sired cattle. Anim Genet. 40:97-100. http://dx.doi.org/10.1111/ j.1365-2052.2008.01790.x

Goddard, M. E. \& B. J. Hayes. 2007. Genomic selection. J. Anim. Breed. Genet. 124:323-330. http://dx.doi.org/10.1111/ j.1439-0388.2007.00702.x

Gorjanc, G., M. A. Cleveland, R. D. Houston \& J. M. Hickey. 2015. Potential of genotyping-by-sequencing for genomic selection in livestock populations. GSE. 47:1-13. http:// dx.doi.org/10.1186/s12711-015-0102-z

Grisolia, A. B., G. T. D'Angelo, L. R. P. Neto, F. Siqueira, \& J. F. Garcia. 2009. Myostatin (GDF8) single nucleotide polymorphisms in Nellore cattle. Genet. Mol. Rese. 8:822-830. http://dx.doi.org/10.4238/vol8-3gmr548

Gupta, S., A. Kumar, S Kumar, Z. F. Bhat, H. R. Hakeem, \& A. P. S. Abrol. 2013. Recent trends in carcass evaluation techniques-a review. J. Meat. Sci. Tech. 1:50-55.

Han, S. H., I. C. Cho, M. S. Ko, E. Y. Kim, S. P. Park, S. S. Lee, \& H. S. Oh. 2012. A promoter polymorphism of MSTN g.2371T $>$ A and its associations with carcass traits in Korean cattle. Mol. Bil. Rep. 39:3767-3772. http://dx.doi. org/10.1007/s11033-011-1153-z 
He, Y. L., Y. H. Wu, F. S. Quan, Y. G. Liu, \& Y. Zhang. 2013. Comparative analysis of myostatin gene and promoter sequences of Qinchuan and Red Angus cattle. Genet. Mol. Res. 12:3398-3406. http://dx.doi.org/10.4238/2013.September.4.6

Illingworth, R. S., U. Gruenewald-Schneider, S. Webb, A. R. W. Kerr, K. D. James, D. J. Turner, C. Smith, D. J. Harrison, R. Andrews, \& A. P. Bird. 2010. Orphan CpG islands identify numerous conserved promoters in the mammalian genome. PLoS Genet. 6:1-15. http://dx.doi.org/10.1371/ journal.pgen.1001134

Kambadur, R., A Bishop, M. S. Salerno, S. McVroskery, \& M. Sharma. 2004. Role of myostatin in muscle growth. P. 297312. In: M. F. W Te Pas, M. E. Everts \& H. P. Haagsman (ed). Muscle development of livestock animals physioly, genetic and meat quality. CABI, USA.

Li, N., Q. Yang, G. W. Ryan, B. Thomas, D. Min, \& D. R. Buel. 2015. Myostatin attenuation in vivo reduces adiposity, but activates adipogenesis. Endocrinology. 157:1-10.

Martojo, H. 2012. Indigenous Bali cattle is most suitable for sustainable small farming in Indonesia. Reprod. Dom. Anim. 47: 10-14. http://dx.doi.org/10.1111/j.14390531.2011.01958.x

Melendez, L. J. \& J. A. Marchello. 2014. The efficacy of ultrasound to determine certain carcass traits in grain-fed beef cattle. Inter. J. Sci. Comm. Hum. 2:145-154.

Miyake, M., S. Hayashi,Y. Taketa, S. Iwasaki, K. Watanabe, S. Ohwada, H. Aso, \& T. Yamaguchi. 2010. Myostatin downregulates the IGF-2 expression via ALK-Smad signaling during myogenesis in cattle. Anim. Sci. Jour. 81:223-229. http://dx.doi.org/10.1111/j.1740-0929.2009.00725.x

Nassiry, M. R., A. Javanmard, \& R. Tohidi. 2009. Application of statistical procedures for analysis of genetic diversity in domestic animal populations. American J. Anim. Vet. Sci. 4:136-141. http://dx.doi.org/10.3844/ajavsp.2009.136.141

Nei, M. \& S. Kumar. 2000. Molecular Evolution and Phylogenetics. Oxford Univ Pr., New York.

Purwantara, B., R. R. Noor, G. Anderson, \& H. RodriguezMartinez. 2012. Banteng and Bali cattle in Indonesia: status and forecasts. Reprod. Dom. Anim. 47:2-6. http:// dx.doi.org/10.1111/j.1439-0531.2011.01956.x

Putri, R., R. Priyanto, A. Gunawan, \& Jakaria. 2015. Association of calpastatin (CAST) gene with growth traits and carcass characteristics in Bali cattle. Med Pet. 38:145-149. http://dx.doi.org/10.5398/medpet.2015.38.3.145
Sarti, F. M., E. Lasagna, S. Ceccobelli, P. Di Lorenzo, F. Filippini, F. Sbarra, \& A. Giontella. 2014. Influence of single nucleotide polymorphism in myostatin and myogenic factor 5 muscle growth-related genes on the performance traits of Marchigiana beef cattle. J. Anim. Sci. 92:3804-3810. http://dx.doi.org/10.2527/jas.2014-7669

SAS Institute Inc. 2008. SAS/STAT® 9.2 User's Guide The GLM Procedure(Book Excerpt). SAS Institute Inc. SAS Campus Drive, Carolina.

Sellner, E. M., J. W. Kim, M. C. Mc Clure, K. H. Taylor, R. D. Schnabel, \& J. F. Taylor. 2007. Board-invited review: applications of genomic information in livestock. J. Anim. Sci. 85:3148-3158. http://dx.doi.org/10.2527/jas.2007-0291

Shibata, M., K. Matsumoto, K. Aikawa, T. Muramoto, S. Fujimura, \& M. Kadowaki. 2006. Gene expression of myostatin during development and regeneration skeletal muscle in Japanese Black Cattle. J. Anim. Sci. 84:2983-2989. http://dx.doi.org/10.2527/jas.2006-118

Silva, S. L., J. U. Tarouco, J. B. S. Ferraz, da C. Gomes, P. R. Leme, \& E. A. Navajas. 2012. Prediction of retail beef yield, trim fat and proportion of high-valued cuts in Nellore cattle using ultrasound live measurements. R. Bras. Zootec. 41:2025-2031. http://dx.doi.org/10.1590/S151635982012000900009

Smith Z. D. \& A. Meissner. 2013. DNA methylation: roles in mammalian development. Nat. Review Genet. 14: 204-220. http://dx.doi.org/10.1038/nrg3354

Sun, W. X., V. Dodson, Z. H. Jiang, S. G. Yu, W. W. Chu, \& J. Chen. 2016. Myostatin inhibits porcine preadipocyte differentiation in vitro. Domes. Anim. Endocri. 55: 25-31. http://dx.doi.org/10.1016/j.domaniend.2015.10.005

Tamura, K., D. Peterson, N. Peterson, G. Stecher, M. Nei, \& S. Kumar. 2011. MEGA5: molecular evolutionary genetics analysis using maximum likelihood. evolutionary distance. and maximum parsimony methods. Mol. Biol. Evol. 28:2731-2739. http://dx.doi.org/10.1093/molbev/msr121

Yeh, F. C., R. C. Yang, \& T. Boyle. 1999. POPGENE 32-Version1.31. Population genetics software. [ 2016 Jan 20]. https://www.ualberta.ca/ fyeh/popgene.pdf.

Zhang, R. F., H. Chen, C. Z. Lei, C. L. Zhang, X. Y. Lan, Y. D. Zhang, H. J. Zhang, B. Bao, H. Niu, \& X. Z. Wang. 2007. Association between polymorphisms of mstn and myf5 genes and growth traits in three chinese cattle breeds. AJAS. 20:1798 - 1804. http://dx.doi.org/10.5713/ajas.2007.1798 